\title{
In vivo disruption of latent HSV by designer endonuclease therapy
}

\author{
Martine Aubert, ${ }^{1}$ Emily A. Madden, ${ }^{1}$ Michelle Loprieno, ${ }^{1}$ Harshana S. DeSilva Feelixge, \\ Laurence Stensland, ${ }^{2}$ Meei-Li Huang, ${ }^{2}$ Alexander L. Greninger, ${ }^{2}$ Pavitra Roychoudhury, ${ }^{1}$ \\ Nixon Niyonzima, ${ }^{1}$ Thuy Nguyen, ${ }^{2}$ Amalia Magaret, ${ }^{1,2}$ Roman Galleto, ${ }^{3}$ Daniel Stone, ${ }^{1}$ \\ and Keith R. Jerome ${ }^{1,2}$ \\ 'Vaccine and Infectious Disease Division, Fred Hutchinson Cancer Research Center, Seattle, Washington, USA. \\ ${ }^{2}$ Department of Laboratory Medicine, University of Washington, Seattle, Washington, USA. \\ ${ }^{3}$ Cellectis Therapeutics, Paris, France.
}

\begin{abstract}
A large portion of the global population carries latent herpes simplex virus (HSV), which can periodically reactivate, resulting in asymptomatic shedding or formation of ulcerative lesions. Current anti-HSV drugs do not eliminate latent virus from sensory neurons where HSV resides, and therefore do not eliminate the risk of transmission or recurrent disease. Here, we report the ability of HSV-specific endonucleases to induce mutations of essential HSV genes both in cultured neurons and in latently infected mice. In neurons, viral genomes are susceptible to endonuclease-mediated mutagenesis, regardless of the time of treatment after HSV infection, suggesting that both HSV lytic and latent forms can be targeted. Mutagenesis frequency after endonuclease exposure can be increased nearly 2-fold by treatment with a histone deacetylase (HDAC) inhibitor. Using a mouse model of latent HSV infection, we demonstrate that a targeted endonuclease can be delivered to viral latency sites via an adeno-associated virus (AAV) vector, where it is able to induce mutation of latent HSV genomes. These data provide the first proof-of-principle to our knowledge for the use of a targeted endonuclease as an antiviral agent to treat an established latent viral infection in vivo.
\end{abstract}

Conflict of interest: The authors have declared that no conflict of interest exists.

Submitted: May 9, 2016

Accepted: August 2, 2016

Published: September 8, 2016

Reference information:

JCI Insight. 2016;1(14):e88468.

doi:10.1172/jci.insight.88468.

\section{Introduction}

Primary infection with herpes simplex virus (HSV) occurs at mucosal surfaces, where the virus accesses nerve endings of sensory neurons. HSV then travels down axons to neuronal cell bodies, where it establishes a stable episomal state and persists for the life of the host. Periodically, episomal HSV can reactivate to produce viral components that travel back down axons and reassemble into replication-competent virus that can infect mucosal epithelial cells, leading to amplification of infectious virus and either asymptomatic viral shedding or development of symptomatic recurrent disease (1). Asymptomatic genital shedding of HSV can be detected on $28 \%$ of days in HSV-2-infected individuals, and symptomatic disease typically recurs, on average, 2.1-6.8 times per year (2-4).

Standard HSV therapy consists of nucleoside analogs such as acyclovir (ACV), valacyclovir, and famciclovir, which shorten the duration of primary and recurrent infections (5-8). If taken for extended periods, they reduce the frequency of symptomatic recurrences (7). However, they do not reduce the frequency of asymptomatic shedding (9) and have only a partial effect on the likelihood of transmission to sexual partners (10). Importantly, the drugs have no effect on the persistent form of HSV, and when treatment is halted, symptomatic recurrence frequencies return to pretreatment levels (4).

The recent development of targeted designer endonucleases (CRISPR/Cas9, zinc finger nucleases, TALENs, and meganucleases [also referred to as homing endonucleases or HEs]) — which cleave and trigger DNA mutagenesis at desired sites with high specificity - raises the possibility of direct disruption of the persistent DNA forms of many viruses (reviewed in ref. 11). For HSV, this approach has been evaluated using in vitro model systems $(12,13)$, but the ability of the episomal form to be disrupted in neurons has not been established. Moreover, for no virus has in vivo disruption of an established persistent infection been demonstrated. Here, we report efficient disruption of persistent HSV in neurons. Furthermore, we demonstrate that in vivo mutagenesis of latent HSV can be achieved in a mouse model of persistent infection. These results support the continued development of endonucleases as antiviral agents. 


\section{Results}

AAV serotype screening for gene delivery to primary murine TG neurons. To test HSV therapeutic enzymes in a relevant system, we first optimized adeno-associated virus (AAV) delivery to primary neuronal cultures established from mouse trigeminal ganglia (TG). A panel of GFP reporter-expressing AAV vectors derived from serotypes $1,5,7,8$, and 9 were screened based on previous reports of neurotropism in different systems (14-18). Cultures treated with AAV1, 7, and 8 had the largest number of $\mathrm{GFP}^{+}$cells, and the majority of $\mathrm{GFP}^{+}$cells were neurons, based on cell morphology (Supplemental Figure 1, A and B; supplemental material available online with this article; doi:10.1172/jci.insight.88468DS1). AAV8 was chosen for subsequent experiments in cultures because it showed a propensity to transduce a lower number of nonneuronal cells (Supplemental Figure 1B), and high-titer stocks were reproducibly generated with this serotype (data not shown).

Promoter screening for gene delivery to primary murine TG neurons. Since self-complementary AAV (scAAV) vectors provide higher levels and faster kinetics of transgene expression, they are preferable to single stranded AAV (ssAAV) vectors for transgene delivery in vitro $(19,20)$. However, scAAV vectors have a payload capacity of $\sim 2.3 \mathrm{~kb}$, requiring use of small promoters. Our AAV serotype screen used a small hybrid cytomegalovirus (CMV)-chicken $\beta$ actin promoter (smCBA). However, this promoter is too large to accommodate an HSV-specific HE due to scAAV size restriction. We therefore tested 2 versions of the immediate early human cytomegalovirus promoter (CMV and short CMV [sCMV]) and the neuron-specific promoter hSyn from the human synapsin I gene. GFP expression from these promoters was compared with the expression obtained with the strong smCBA promoter, in the context of both scAAV1 and scAAV8 vectors. For all promoters, GFP transgene expression was detected by 3 days after transduction, plateaued by day 14, and was maintained throughout the 21-day duration of the experiment (Supplemental Figure 1, C and D). The smCBA promoter produced the highest levels of transgene expression. Both CMV-derived promoters produced higher levels of GFP expression than hSyn, with sCMV being slightly better than CMV (Supplemental Figure 1, E and F). Thus, the sCMV promoter was chosen for scAAV constructs for further testing in TG neuronal cultures.

Inhibition of active HSV replication in primary murine TG neurons. We previously showed that levels of HSV-specific, HE-directed mutagenesis are higher during lytic compared with latent HSV infection in primary fibroblasts (13). We therefore first evaluated targeted mutagenesis during lytic infection of primary neurons. Two I-CreI-derived HSV-specific HEs, HSV1m5 or HSV1m8, targeting sequences in the essential genes $U_{L} 19$ (encoding the major virion protein VP5) or $U_{L} 30$ (encoding the DNA polymerase catalytic subunit) were used (Figure 1, A and B). These enzymes were selected from a proprietary collection of over 30,000 engineered endonucleases, derived from I-CreI in a combinatorial process. They were selected based on their activity against HSV-1 genomes and their target site location in viral essential genes $(12,13)$. Additionally, as a control, we used nonviral enzyme 1 (NV1), which is also derived from I-CreI but recognizes a target site in the human $R A G-1$ gene (21). We previously showed that HE-directed mutagenesis in primary cells is substantially increased by coexpression of Trex2, a 3'-5' exonuclease (13). To codeliver the HSV-specific HE and Trex2, two separate scAAV8 vectors were required, and we confirmed that high levels of cotransduction could be achieved in primary TG neuronal cultures using AAV (Figure 1C). Cultured primary neurons were first cotransduced with scAAV8 vectors expressing HE (HSV1m5 or HSV1m8) or a control fluorescent reporter (eGFP or mCherry) and Trex2 (Figure 1D), followed by infection with HSV-1. Production of HSV was then measured in supernatants at 4 days after HSV infection, revealing a $>50 \%$ reduction in HSV-1 production from the HE/Trex2-treated cells compared with control cells (Figure 1E, $P$ $<0.01$ ). The T7 Endonuclease 1 (T7E1) assay indicated mutation of HSV in treated but not control samples (Figure 1F); see complete unedited blots in the supplemental material. The presence of mutations was confirmed by clonal sequencing (Figure $1 G$ ), which revealed small deletions consistent with those previously observed with these enzymes $(12,13)$. In a separate experiment with a slightly different time course, we observed a one-log reduction of virus titers in HSV1m5/Trex2-treated cells compared with controls (Supplemental Figure 2, A and B). The presence of mutations in the HE target site of these treated cells in this second experiment was again demonstrated using the T7E1 assay (Supplemental Figure 2C); see complete unedited blots in the supplemental material.

Targeted mutagenesis of HSV genomes in neurons from HSV-infected mice. We next determined whether HSV genomes present in infected TG neurons after in vivo viral infection were susceptible to HE-directed mutagenesis. At 7 days after HSV infection, acute viral replication in the TG has largely subsided, but 


\section{A Schematic of the HSV genome}
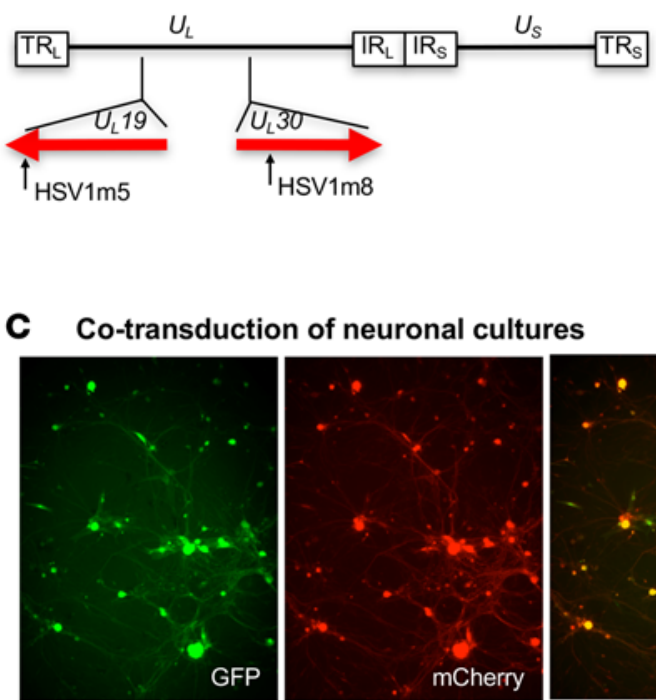

D Experimental timeline

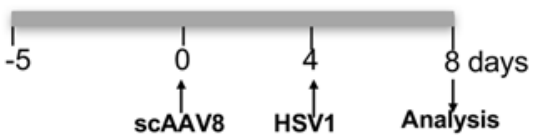

F Detection of mutation by T7E1
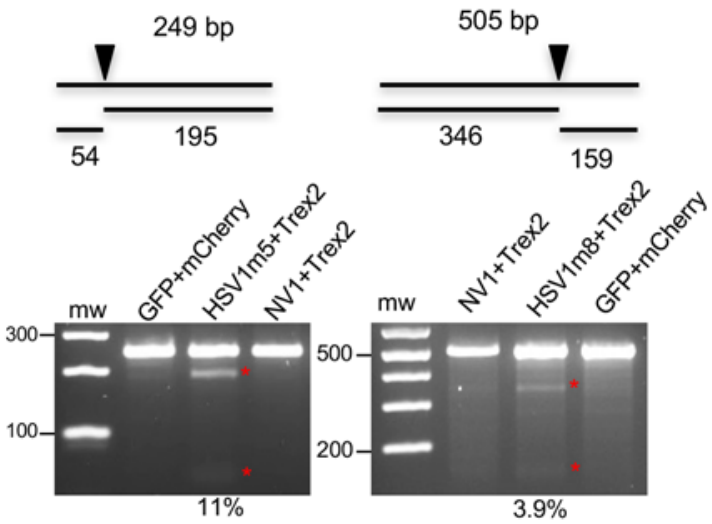

\section{B HSV-specific target sequences}

HSV1m5 target site

5'-ATAAACTCACACAC'GGCGTCCTGG

3'-TATTTGAGTGTGTGCCGCAGGACC $\uparrow$

HSV1m8 target site

5'-CCGCTCTGTTTTACCCGGTCTACG

3'-GGCGAGACAAAATGGCGCAGATGC

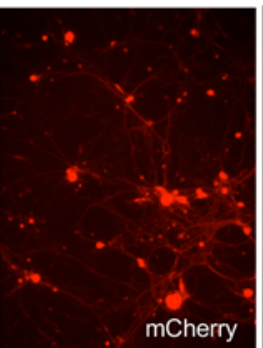

mCherry

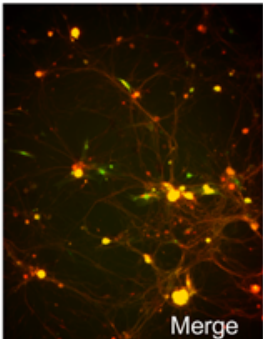

E Virus production in treated cells

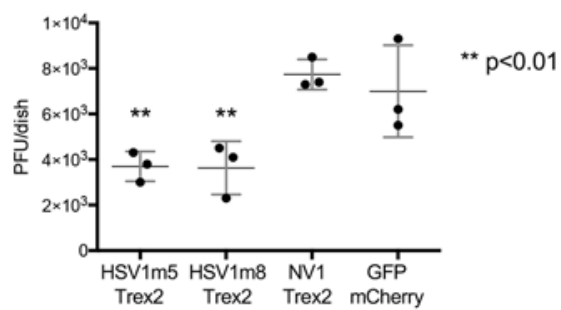

G Sequence analysis of targeted sites

HSV $1 \mathrm{~m} 5$

ATAAACTCACACACGGCGTCCTGG wt ATAAACTCACAC--GGCGTCCTGG $\triangle 2 \mathrm{bp}$ ATAAACTCACA---GGCGTCCTGG $\triangle 3 \mathrm{bp}$ ATAAACTCAC----GGCGTCCTGG $\triangle 4 \mathrm{bp}$ HSV1m8

CCGCTCTGTTTTACCGCGTCTACG wt CCGCTCTGTTT-ACCGCGTCTACG $\triangle 1 \mathrm{bp}$ CCGCTCTGTTT--CCGCGTCTACG $\triangle 2 \mathrm{bp}$ CCGCTCTGTT----CGCGTCTACG $\Delta 4 \mathrm{bp}$ CCGCTCTGTTT---------TACG $\triangle 9 \mathrm{bp}$

Figure 1. Impact of HSV-specific HE exposure on HSV replication in neuronal cultures. (A) Schematic representation of the HSV-1 genome. The long terminal and internal repeats (TR and IR $)$, and the internal and terminal short repeats $\left(T R_{S}\right.$ and $\left.I R_{S}\right)$, bordering the unique long $\left(U_{L}\right)$ and unique short $\left(U_{S}\right)$ regions are shown. The location of the target sequences recognized by HSV1m5 in the $U_{L} 19$ gene and HSV1m8 in the $U_{L} 30$ gene are indicated. (B) The sequences rec ognized by HSV1m5 and HSV1m8 are shown. The 4 nucleotides constituting the $3^{\prime}$ overhang generated by HE cleavage are bold and underlined. Arrows indicate enzyme target or cleavage sites. (C) Fluorescence images of neuronal cultures taken at 7 days after cotransduction with scAAV8-sCMV-GFP and scAAV8-sCMV-mCherry at an MOI of $1 \times 10^{6} \mathrm{vg} / \mathrm{AAV} /$ neuron. Representative GFP, mCherry, merged GFP/mCherry, and phase images are shown. Magnification 100×. (D) Schematic of the experimental timeline. Triplicate neuronal cultures were established from dissociated murine TGs and cotransduced with 2 scAAV8 vectors expressing HSV1m5 and Trex2, HSV1m8 and Trex2, NV1 and Trex2, or GFP and mCherry at a $\mathrm{MOI}$ of $1 \times 10^{6} \mathrm{vg} / \mathrm{AAV} /$ neuron. At 4 days after AAV transduction, cultures were infected with HSV- $\mathrm{F} \Delta U_{5} 5$ at an $\mathrm{MOI}$ of $1 \mathrm{PFU} /$ neuron for 4 additional days. (E) Virion release in culture media was quantified by plaque assay titration. The mean \pm SD of triplicate wells is indicated. The $P$ value was calculated using a 1-sided $t$ test. (F) Mutagenic event detection by T7E1 assay. Schematic representation of PCR amplicon with full-size and T7 endonuclease cleavage product sizes indicative of HSV-specific HE cleavage and mutagenesis. The arrow indicates the location of the HE target site in the PCR product. The HSV regions containing the target site were PCR amplified from total genomic DNA obtained from pooled triplicate dishes and subjected to T7E1 digestion before separation on a 3\% agarose gel. mw, molecular weight size ladder; red asterisks indicate cleavage products. The relative mutation frequency was determined using Image). (G) Target site-containing PCR amplicons were cloned and sequenced from individual bacterial colonies. $\Delta$, deletion. Bold indicates the 4 nucleotides constituting the $3^{\prime}$ overhang generated upon HE target site cleavage. 
Table 1. Sequence analysis of HSV1m5 $\left(U_{L} 19\right)$ and HSV1m8 $\left(U_{L} 30\right)$ target sites

\begin{tabular}{|c|c|c|c|c|}
\hline & Gene & Acute & Late/Acute & Latent \\
\hline \multirow{2}{*}{ Number of mutants } & $U_{L} 19$ & 5 & 4 & 7 \\
\hline & $U_{L} 30$ & 1 & 6 & 4 \\
\hline \multirow{2}{*}{ Number of clones screened } & $U_{L} 19$ & 82 & 89 & 92 \\
\hline & $U_{L} 30$ & 88 & 91 & 90 \\
\hline \multirow{2}{*}{$\%$ mutation } & $U_{L} 19$ & $6.1 \%$ & $4.5 \%$ & $7.6 \%$ \\
\hline & $U_{L}^{L} 30$ & $1.1 \%$ & $6.6 \%$ & $4.4 \%$ \\
\hline \multirow{2}{*}{ Number and type of mutation } & $U_{L} 19$ & $\begin{array}{l}1 \times \Delta 1 \\
3 \times \Delta 2 \\
1 \times \Delta 3\end{array}$ & $4 \times \Delta 2$ & $\begin{array}{l}6 \times \Delta 2 \\
1 \times \Delta 5\end{array}$ \\
\hline & $U_{L} 30$ & $1 \times \Delta 1$ & $\begin{array}{c}2 \times \Delta 1 \\
3 \times \Delta 2 \\
1 \times+1\end{array}$ & $\begin{array}{l}2 \times \Delta 1 \\
2 \times \Delta 2\end{array}$ \\
\hline
\end{tabular}

$\Delta$, deletion.

latency is not yet fully established $(22,23)$. As HSV infection progresses, heterochromatin marks increase on histones associated with the viral genome (24). This could result in decreased HE accessibility to HSV genomes. Therefore, mutagenesis of viral genomes was compared in neuronal cultures from mouse TG obtained at 7 (acute phase), 14 (late acute/early latent phase), and 32 (latent phase) days after HSV-1 infection (Figure 2A). For the experiment duration, ACV was included in the culture medium to prevent HSV reactivation (Figure $2 \mathrm{~B}$ ). The levels of target-site mutations observed in treated cells ranged from $4.5 \%-7.6 \%$ for HSV1m5/Trex2 to $1.1 \%-6.6 \%$ for HSV1m8/Trex2 (Table 1). Mutation levels did not vary according to the time the TGs were collected, suggesting that HSV-1 genomes in TG neurons were similarly susceptible to HE-directed mutagenesis during all phases of infection.

HDACi treatment increases targeted mutagenesis of HSV in TG neurons in vitro. Previously, we showed that HE-directed mutagenesis of viral genomes could be increased by exposure to histone deacetylase inhibitors (HDACi) in a fibroblast model of HSV latency (13). Therefore, we investigated whether mutagenic event frequencies could also be increased in HE-exposed HSV-infected primary neurons treated with trichostatin A (TSA) (Figure 2C). In agreement with our previous results, TSA treatment increased mutation of HSV genomes by approximately 2-fold: $5.4 \%-8.9 \%$ for HSV $1 \mathrm{~m} 5$ and $4.5 \%-8.1 \%$ for HSV1m8 (Figure 2, D and E); see complete unedited blots in the supplemental material.

Targeted mutagenesis of latent HSV-1 genomes in vivo. We next asked whether latent HSV could be a target for gene disruption in vivo. HSV-specific HEs were delivered by intradermal whiskerpad injection of AAV1 vectors, which efficiently transduces sensory TG neurons in vivo (Dang et al., unpublished). Mice were infected with HSV-1 by ocular inoculation, and at 32 days after infection, mice were injected with 1 $\times 10^{12}$ vector genomes of ssAAV1 vectors expressing HSV1m5 plus Trex 2 or the control nonviral HE NV1 plus Trex2. At 30 days after AAV1 delivery, TGs were analyzed for levels of HSV-1 genomes, AAV vector genomes, and mutagenesis of HSV genomes (Figure 3, A and B).

Table 2. HSV1m5 target site analysis by NGS, clonal sequencing, and T7E1 assay

\begin{tabular}{|c|c|c|c|c|c|}
\hline Mouse identification number & \multicolumn{3}{|c|}{ Next-Generation Sequencing } & \multicolumn{2}{|c|}{ Other assays } \\
\hline 1 & PBS & $\Delta 1, \Delta 2$ & $0.04 \%$ & N/A & - \\
\hline 8 & HSV1m5/Trex2 & $\Delta 1, \Delta 2, \Delta 4, \Delta 8$ & $2.05 \%$ & $1.1 \%$ & $1.5 \%$ \\
\hline 9 & HSV1m5/Trex2 & $\Delta 1, \Delta 2$ & $0.07 \%$ & $0 \%$ & - \\
\hline 10 & HSV1m5/Trex2 & $\begin{array}{c}\Delta 1, \Delta 2, \Delta 4, \Delta 6, \Delta 8 \\
\Delta 11, \Delta 14\end{array}$ & $3.92 \%$ & $2.2 \%$ & $1.4 \%$ \\
\hline
\end{tabular}

$\Delta$, deletion; -, no detectable cleavage products. 


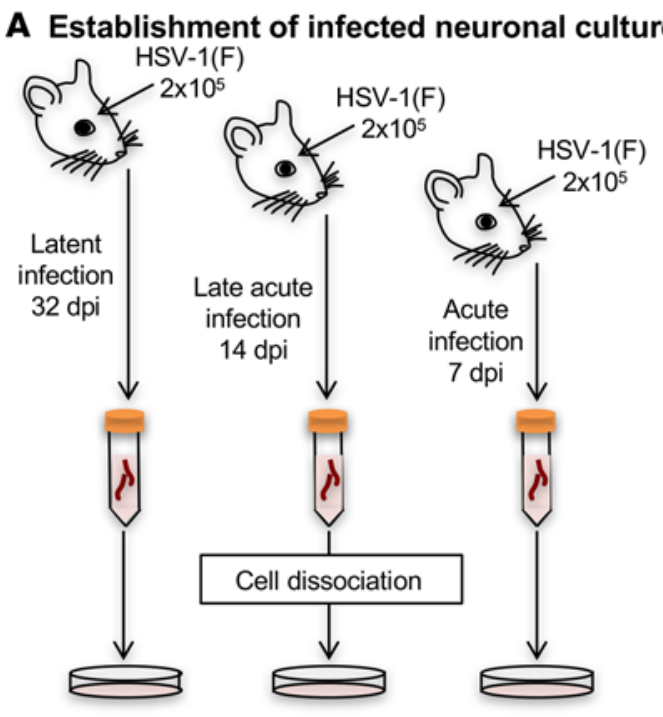

B Schematic of experimental timeline

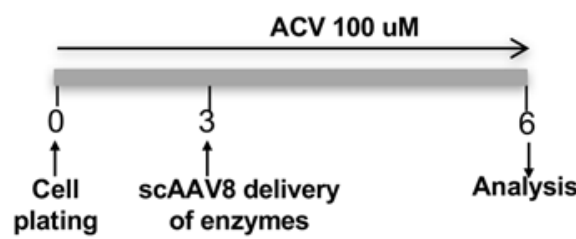

Schematic of experimental timeline

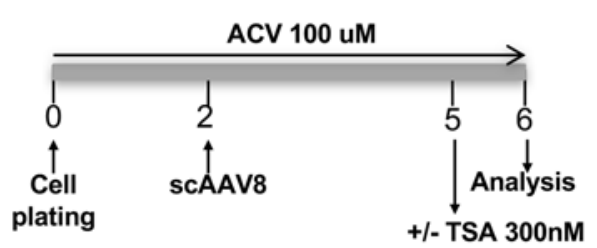

D Detection of mutation T7E1 assay

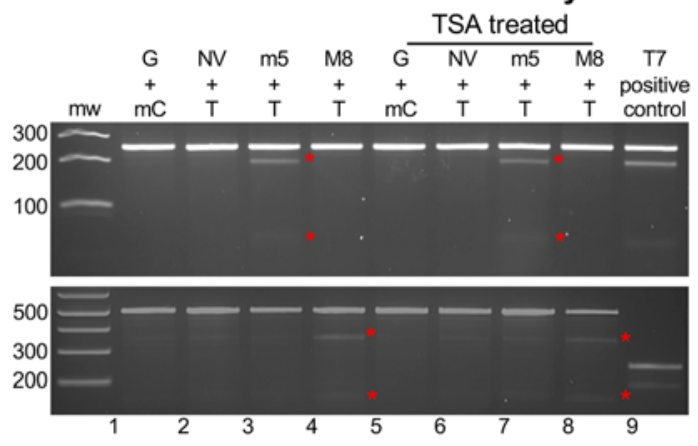

E Sequence analysis of targeted sites

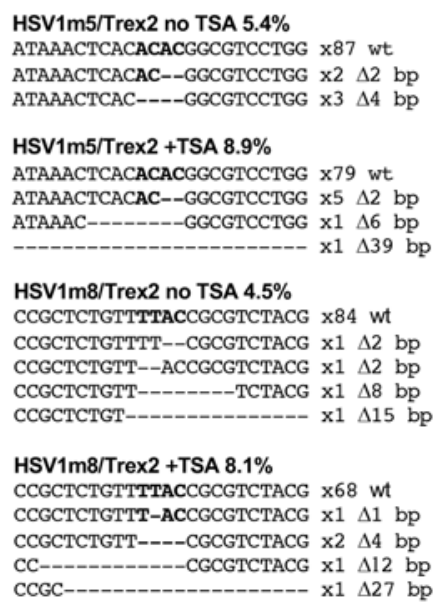

Figure 2. In vitro-targeted mutagenesis in neurons isolated from HSV-infected mice. (A) Schematic of neuronal culture generation. Five mice were infected with $2 \times 10^{5} \mathrm{PFU}$ of HSV-1(F) in the right eye following corneal scarification for each time point and left for 7, 14, or 32 days prior to collection of the right TGs. After tissue dissociation by enzymatic digestion, neuronal cultures were established, and cells were cultured in medium supplemented with $100 \mu \mathrm{M}$ ACV. (B) Experimental timeline. Cells were cotransduced in quadruplicate with either scAAV8-sCMV-HSV1m5 and scAAV8-sCMV-Trex2 or scAAV8-sCMV-HSV1m8 and scAAV8-sCMV-Trex2 at an MOI of $1 \times 10^{6} \mathrm{vg} / \mathrm{AAV} /$ neuron for 3 days prior to analysis. (C) Timeline for the evaluation of the effect of TSA. Nine mice were infected with $2 \times 10^{5} \mathrm{PFU}$ in the right eye following corneal scarification and, 7 days later, the right TGs were collected. After tissue dissociation by enzymatic digestion, neuronal cultures were established and cells were cultured in medium supplemented with $100 \mu \mathrm{M}$ ACV. Eight wells per condition were cotransduced with scAAV8 vectors expressing HSV1m5 and Trex2, HSV1m8 and Trex2, NV1 and Trex2, or GFP and mCherry at an MOI of $1 \times 10^{6} \mathrm{vg} / \mathrm{AAV} /$ neuron for 3 days, after which 4 wells were left untreated while the other 4 were treated with $300 \mathrm{nM}$ TSA for 1 day prior to analysis. (D) Mutagenic event detection by T7E1 assay. The HSV regions containing the target site were PCR amplified from total genomic DNA obtained from pooled quadruplicate wells, subjected to T7E1 digest, and separated on a $3 \%$ agarose gel. mw, molecular weight size ladder; red asterisks indicate cleavage products. G, GFP; NV, NV1; mC, mCherry; T, Trex2; M8, HSV1m8, m5, HSV1m5; T7, T7E1. (E) HSV target sequences from enzyme-treated neurons with and without TSA. ACV, Acyclovir.

AAV genome quantification in the TG by droplet digital PCR (ddPCR) revealed an average of $2 \times 10^{4}$ and $8 \times 10^{3} \mathrm{AAV}$ vector genomes/TG in NV1/Trex2- and HSV1m5/Trex2-treated mice, respectively (Figure $3 \mathrm{C}$ ). We next assessed the presence of mutation in HSV genomes using the T7E1 assay, which indicated that at least 2 of 4 samples from mice treated with HSV1m5/Trex 2 contained mutations (Figure 3D, lane 8 and 10); see complete unedited blots in the supplemental material. Clonal sequencing revealed mutation 
A Experimental timeline

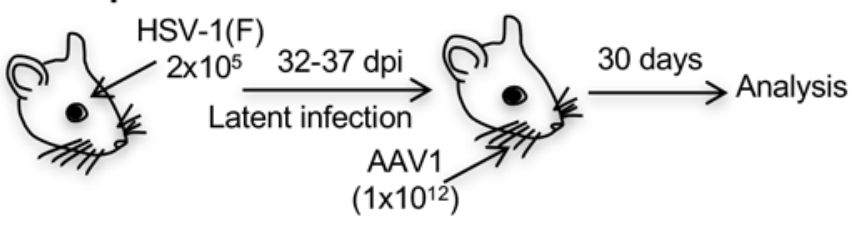

C AAV genome quantification

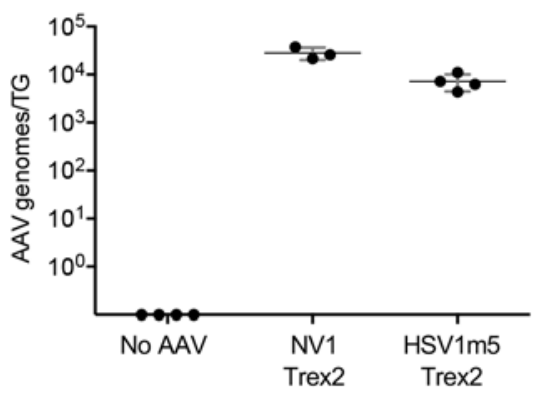

E Clonal sequencing

Mouse \#8 - 1.1\%

ATAAACTCACACACGGCGTCCTGG $x 89$ wt ATAAACTCAC----GGCGTCCTGG x1 $\Delta 4$ bp

Mouse \#10 - 2.2\%

ATAAACTCACACACGGCGTCCTGG $x 88$ wt

ATAAACTCACAC--GGCGTCCTGG $\times 1 \quad \Delta 2$ bp

ATAAACTCAC----GGCGTCCTGG $\times 1 \quad \Delta 4$ bp

\section{B AAV constructs}

\begin{tabular}{|c|c|c|c|c|c|c|}
\hline ITR & smCBA & NV1 & $2 \mathrm{~A}$ & Trex2 & $2 \mathrm{~A}$ & mCherry ITR \\
\hline ITR & smCBA & HSV1m5 & $2 \mathrm{~A}$ & Trex2 & $2 \mathrm{~A}$ & \begin{tabular}{l|l} 
mCherry ITR
\end{tabular} \\
\hline ITR & smCBA & HSV1m8 & $2 \mathrm{~A}$ & Trex2 & $2 \mathrm{~A}$ & mCherry \\
\hline
\end{tabular}

D T7 endonuclease assay analysis

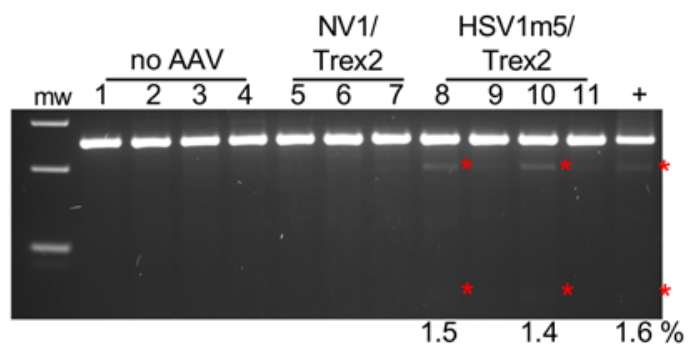

\section{F HSV genome quantification}

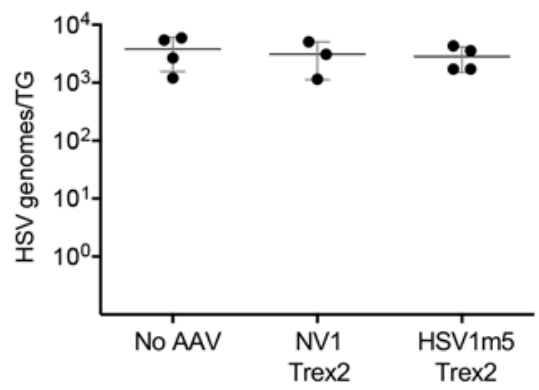

Figure 3. HE-directed mutagenesis of latent HSV in vivo. (A) Experimental timeline. Mice were infected with $2 \times 10^{5} \mathrm{PFU} H S V-1(\mathrm{~F})$ in the right eye following corneal scarification and, 32-37 days later, were injected in the right whiskerpad with $1 \times 10^{12}$ vector genomes of ssAAV1-smCBA-HSV1m5-Trex2-mCherry or ssAAV1-smCBA-NV1-Trex2-mCherry. Analysis was performed at 30 days after AAV exposure. (B) Schematic representation of the ssAAV constructs used here and Figure 6. (C) Levels of AAV genomes were quantified by ddPCR in right (ipsilateral) TCs from infected mice. Mean \pm SD are indicated. (D) Mutagenic event detection by T7E1 assay. The HSV regions containing the target site were PCR amplified from total genomic DNA obtained from the right TC. Products were subjected to T7E1 digestion and separated on a 3\% agarose gel. mw, molecular weight size ladder; red asterisks indicate cleavage products. (E) Clonal sequencing of PCR amplicons from TC of treated animals. (F) Levels of latent HSV genomes were quantified by ddPCR in right (ipsilateral) TGs from infected mice. Mean \pm SD are indicated.

rates of $1.1 \%$ and $2.2 \%$ in the 2 samples showing cleavage products by T7E1 assay. Small deletions of 2 bp or $4 \mathrm{bp}$ were found in the HE target site from these 2 samples, while no mutations were detected in the other HSV1m5/Trex2-treated mice using this method (Figure 3E). Mutation rates in the HSV genomes of HSV1m5/Trex2-treated animals were further analyzed using next generation sequencing (NGS), confirming mutation rates of $2 \%-4 \%$ in 3 of 4 mice treated with HSV1m5/Trex2, with the observed mutations consisting of small deletions of 1-14 bp (Table 2, and Supplemental Table 1). Finally, HSV genome quantification in the TG by ddPCR showed similar levels of latent HSV genomes across all treated and untreated animals, suggesting that HSV-specific HEs did not reduce total latent HSV viral loads (Figure 3F).

Histological and immunohistochemical analysis was performed using TGs from a subset of HSV1 naive control animals either left untreated (PBS) or injected with $1 \times 10^{12}$ AAV1 vector genomes of HSV1 5 /Trex 2 or NV1/Trex 2 in the right whiskerpad. AAV-derived expression of the mCherry reporter was detected by IHC in TGs collected 30 days after AAV injection, and mCherry ${ }^{+}$neurons were detected in TG sections from both HSV1m5/Trex2- and NV1/Trex2-treated mice (Figure 4A). No evidence of neuronal cell death or difference in overall TG morphologies was observed in TG sections from 
Table 3. NGS analysis of the HSV1m5 target site in HSV genomes

\begin{tabular}{|c|c|c|c|c|}
\hline Mouse identification number & Treatment & $\%$ mutation & $\%$ deletions & $\%$ insertions \\
\hline 1 & PBS & 0.03 & 0.03 & 0 \\
\hline 2 & NV1/Trex2 & 0.16 & 0.13 & 0.03 \\
\hline 3 & HSV1m5/Trex2 & 0.06 & 0.05 & 0.01 \\
\hline 4 & HSV1m5/Trex2 & 0.19 & 0.19 & 0 \\
\hline 5 & HSV1m5/Trex2 & 2.05 & 1.88 & 0.17 \\
\hline 6 & HSV1m5/Trex2 & 1.79 & 1.77 & 0.02 \\
\hline
\end{tabular}

treated vs. control (PBS) mice (Figure 4B). Furthermore, no inflammation was observed in TG from NV1/Trex2-treated, HSV1m5/Trex2-treated, or untreated (PBS) animals.

To confirm our in vivo mutagenesis results and extend them to another HSV-specific HE (HSV1m8), a second set of HSV-infected mice were exposed for 30 days to one of the HSV-specific HEs (HSV1m5 or HSV1m8) in combination with Trex2 (Figure 3, A and B). AAV genome quantification showed an average of $7.5 \times 10^{3}$ and $4.6 \times 10^{3}$ vector genomes/TG in HSV1m5/Trex2- and HSV1m8/Trex2-treated mice, respectively (Figure 5A), consistent with our previous results. Evaluation of HSV mutation using the T7E1 assay indicated the presence of target site mutations in viral genomes from 1 of 4 and 3 of 4 mice treated with HSV1m5/trex2 or HSV1m8/Trex2, respectively (Figure 5B); see complete unedited blots in the supplemental material. Deep sequencing of the same samples confirmed these results, with mutation rates of $1.8 \%$ and $2 \%$, respectively, in the 2 mice treated with HSV $1 \mathrm{~m} 5 / \mathrm{Trex} 2$, and $1 \%-1.8 \%$ in the 3 mice treated with HSV1m8/Trex2. The large majority of the mutations detected in the HSV target sites were small deletions, and only a small proportion consisted of insertions (Table 3 and 4). Finally, HSV genomes were detected at similar levels by ddPCR in TG from all latently infected mice, with the exception of 2 animals that had undetectable levels (Figure 5C).

HE off-target activity analysis. To determine whether the HSV-specific HEs used in this study had detectable off-target activity in treated animals, we selected up to 10 potential off-target sites contained in the mouse genome with 6 or fewer mismatches from the HE sites (Supplemental Table 2) using Prognos. We then performed NGS on PCR amplicons of the regions containing the off-target sites in TG genomic DNA extracted from the mice showing the highest on-target activity with HSV1m5/Trex2 (mouse 5) or from mice showing the highest on-target activity with HSV1m8/Trex2 (mouse 10), along with control animals (PBS [mouse 1] and NV1/Trex2 [mouse 2]), from the experiment presented in Figure 5B and Tables 3 and 4. The levels of mutation detected at the off-target sequences in DNA samples from treated animals were low and were similar in frequency and distribution across the sites to the levels observed in control animals (Figure 6).

$H S V$ reactivation in $T G$ from $H E$-treated mice. As noted above, reproducible gene disruption was observed in $1 \%-4 \%$ of latent HSV genomes in treated mice. To evaluate whether the targeted endonucleases had an impact on viral reactivation in treated animals, latently infected mice were either left untreated (PBS) or exposed for 31 days to either HSV1m5 or the control enzyme NV1, in combination with Trex2, by whiskerpad injection of AAV1 vector (Figure 7A). Then, individual TGs were explanted into

Table 4. NGS analysis of the HSV1m8 target site in HSV genomes

\begin{tabular}{|c|c|c|c|c|}
\hline Mouse identification number & Treatment & $\%$ mutation & $\%$ deletions & $\%$ insertions \\
\hline 1 & PBS & 0.16 & 0.08 & 0.08 \\
\hline 2 & NV1/Trex2 & 0.16 & 0.09 & 0.07 \\
\hline 7 & HSV1m8/Trex2 & 0.32 & 0.24 & 0.08 \\
\hline 8 & HSV1m8/Trex2 & 1.27 & 1.07 & 0.20 \\
\hline 9 & HSV1m8/Trex2 & 1.04 & 0.71 & 0.33 \\
\hline 10 & HSV1m8/Trex2 & 1.82 & 1.60 & 0.22 \\
\hline
\end{tabular}


A IHC staining (NeuN and mCherry) NeuN
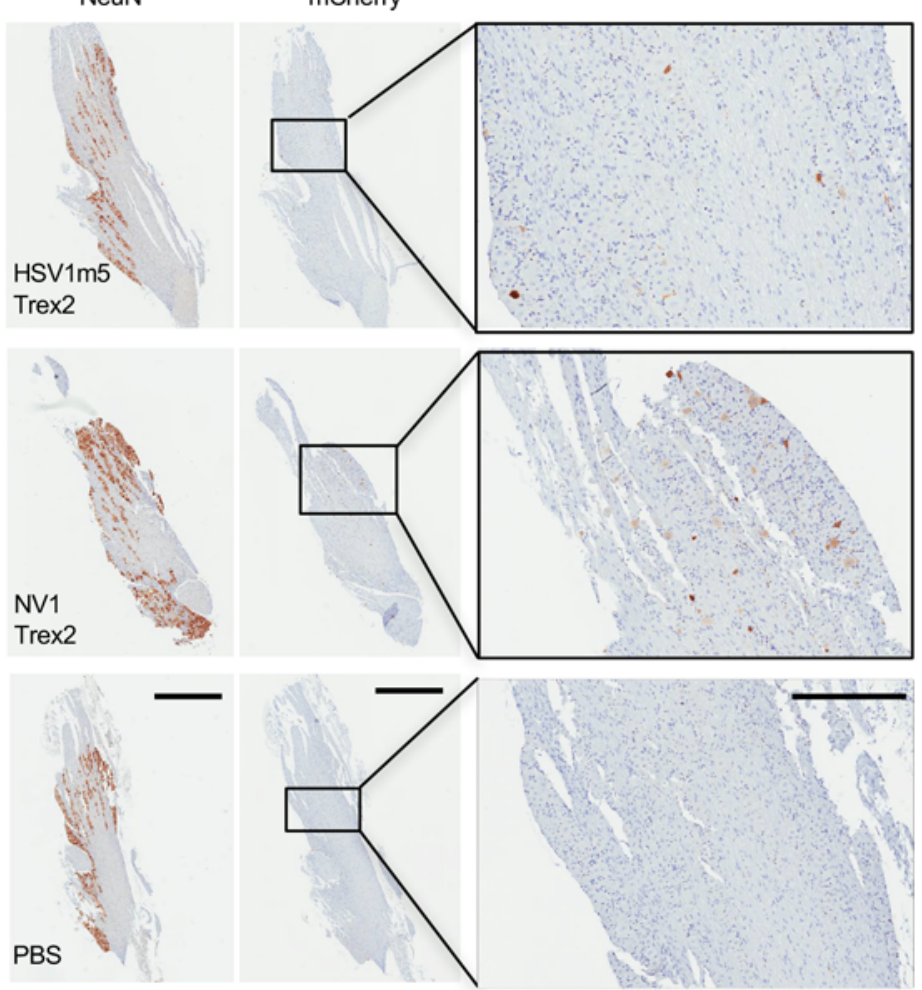

\section{B Histological staining (H\&E)}
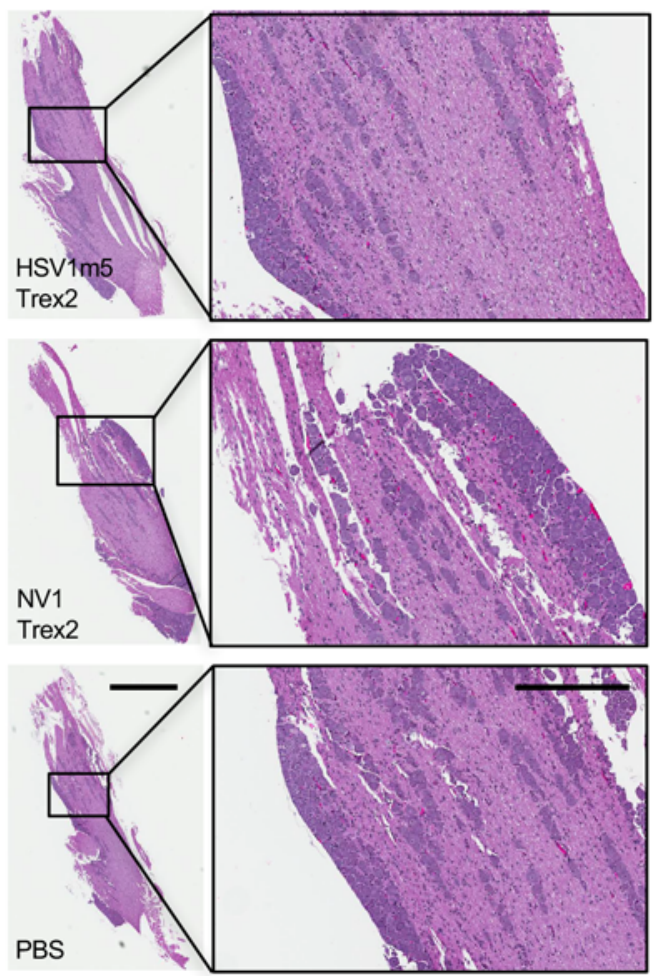

Figure 4. IHC and histological staining of TC from mice exposed to HSV-specific HE. (A) IHC staining for NeuN and mCherry. Left and middle panels scale bar: $1 \mathrm{~mm}$; right panels scale bar: $300 \mu \mathrm{M}$. (B) H\&E staining of TGs from naive control mice injected with either $1 \times 10^{12}$ ssAAV1-smCBA-NV1-Trex2-mCherry, ssAAV1-smCBA-NV1-Trex2-mCherry, or $50 \mu \mathrm{l} \mathrm{PBS.} \mathrm{Left} \mathrm{panels} \mathrm{scale} \mathrm{bar:} 1 \mathrm{~mm}$; right panels scale bar: $300 \mu \mathrm{M}$

culture wells containing Vero cell monolayers. Culture medium was collected daily for 7 days and used for quantitative PCR (qPCR) quantification of HSV production from the explanted ganglia, as well as for plaque assay on indicator Vero cells, to detect the release of infectious viruses (Figure 7A). TG from 3 animals in each group (controls or HSV1 5 /Trex2-treated mice) did not have reactivable HSV. In the remaining animals, qPCR analysis showed the expected increasing levels of HSV DNA over the 7-day period, with several of the HSV1m5/Trex2-treated animals showing delayed HSV outgrowth. While the estimated median time to positivity (set at 1,000 genome copies) was 2 days for PBS- and NV1/ Trex2-treated mice and 4 days for HSV1m5/Trex2-treated animals (Figure 7B), the difference did not reach statistical significance $(P=0.12)$ when evaluated by the log-rank test. Next, release of infectious viruses in the culture supernatants was assessed by plaque assay. Presence of infectious viruses in TG explant culture supernatants was detected starting at day 2 (NV1/Trex2-treated group) or 3 (PBS- and HSV1m5/Trex2-treated groups) after TG explant. While all the TG with reactivable HSV in the 3 groups produced infectious viruses by day 7 after explant, a delay was again observed in HSV1m5/Trex2-treated animals compared with the 2 control groups. The estimated median time to culture positivity for PBSand NV1/Trex2-treated mice was 3 days and 4 days for HSV1m5/Trex2-treated animals (Figure 7C). However, statistical analysis of the time to TG explant culture positivity revealed that this difference was not statistically significant $(P=0.2)$ when evaluated using a log-rank test.

\section{Discussion}

In this paper, we demonstrate that a targeted endonuclease can be delivered to TG of HSV-infected mice, where it is able to induce mutation of latent HSV genomes. To our knowledge, this is the first demonstration of an endonuclease treatment for an established latent viral infection in vivo. As such, it offers important proof-of-principle for this therapeutic approach and raises the conceptual possibility of cure for 
A AAV genome quantification

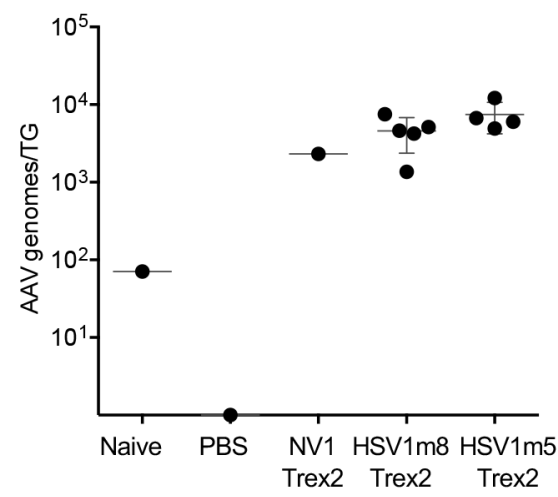

C HSV genome quantification

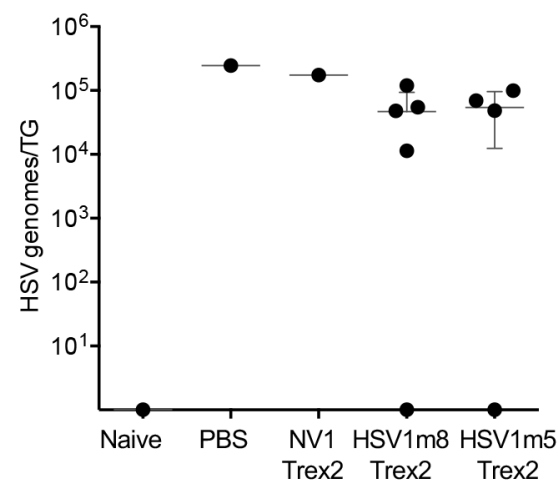

B T7 endonuclease assay analysis

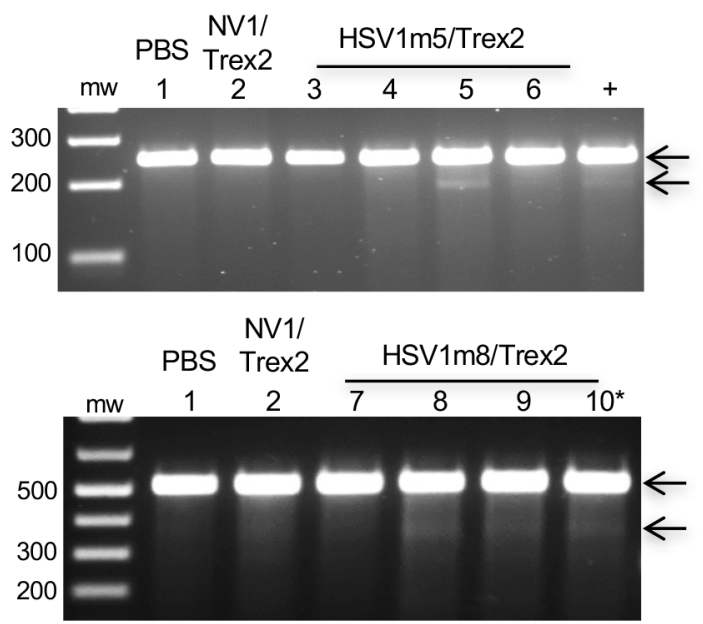

Figure 5. HE-directed mutagenesis of latent HSV in vivo. (A) Levels of AAV genomes were quantified by ddPCR in right (ipsilateral) TGs from infected and naive mice. Mean \pm SD are indicated. (B) Mutagenic event detection by T7E1 assay. The HSV regions containing the target site for HSV1m5 (top panel) or HSV1m8 (bottom panel) were PCR amplified from total genomic DNA obtained from the right (ipsilateral) TC. Products were subjected to T7E1 digestion and separated on a $3 \%$ (HSV1m5) or 1\% (HSV1m8) agarose gel. mw, molecular weight size ladder. (C) Levels of latent HSV genomes were quantified by ddPCR in right (ipsilateral) TGs from infected and naive mice. Mean \pm SD are indicated.

otherwise incurable chronic and latent viral infections.

Several aspects of our work represent important progress toward the use of endonucleases as an antiHSV therapy. First, we demonstrated the introduction of endonuclease-induced mutations in latent HSV genomes in neurons, the biologically relevant cell type. Previously, we and others showed that HSV infection is inhibited in the presence of HSV-specific endonucleases in vitro $(12,13,25)$. We further demonstrated in vitro that HSV-specific HEs could mediate mutagenesis of latent HSV and limit subsequent reactivation (13). However, none of these previous studies were done in neuronal cells $(12,13,25)$, and the demonstration that endonucleases can also mutate HSV in sensory neurons, the relevant cell type for HSV latency, provides support for evaluating this approach in vivo.

Second, our work makes the important point that the latent form of HSV can be a viable endonuclease target. During latency, the HSV genome is associated with histones, which harbor modifications characteristic of transcriptionally inactive DNA or heterochromatin. These modifications confer a closed conformation to the DNA, rendering it less accessible to transcription factors and/or activators and leading to repression of gene expression (26). The modifications may also reduce the access of endonucleases to their target site and decrease the efficiency of HE-directed mutagenesis. A recent study of in vivo accumulation of heterochromatin histone modifications on HSV genome showed that $\mathrm{H} 3$ histones were associated with viral gene promoters by 7 days after infection (dpi), and facultative heterochromatin mark H3K27me3 was low at $7 \mathrm{dpi}$ but increased dramatically by $14 \mathrm{dpi}$ (24). We therefore evaluated HE-directed mutagenesis of HSV genomes in neuronal cultures established from TG - collected at 7, 14, or $32 \mathrm{dpi}-$ to determine whether chromatinization and accumulation of heterochromatin marks during establishment of HSV latency had any impact on the targeted mutagenesis efficiency. Mutation levels did not vary according to the time the TGs were collected, suggesting that HSV-1 genomes in TG neurons were similarly susceptible to HE-directed mutagenesis throughout the phases of infection. In vitro, HDACi such as TSA can reactivate latent HSV (27), and our previous in vitro studies revealed that TSA or other HDACi treatment prior to exposure to HSV-specific HEs resulted in an increase of targeted mutagenesis by at least 2-fold (13). Similarly, in this report we showed that the frequency of HE-directed mutagenesis was increased by almost 


\section{A NGS analysis of On and Off target activity of HSV1m8}

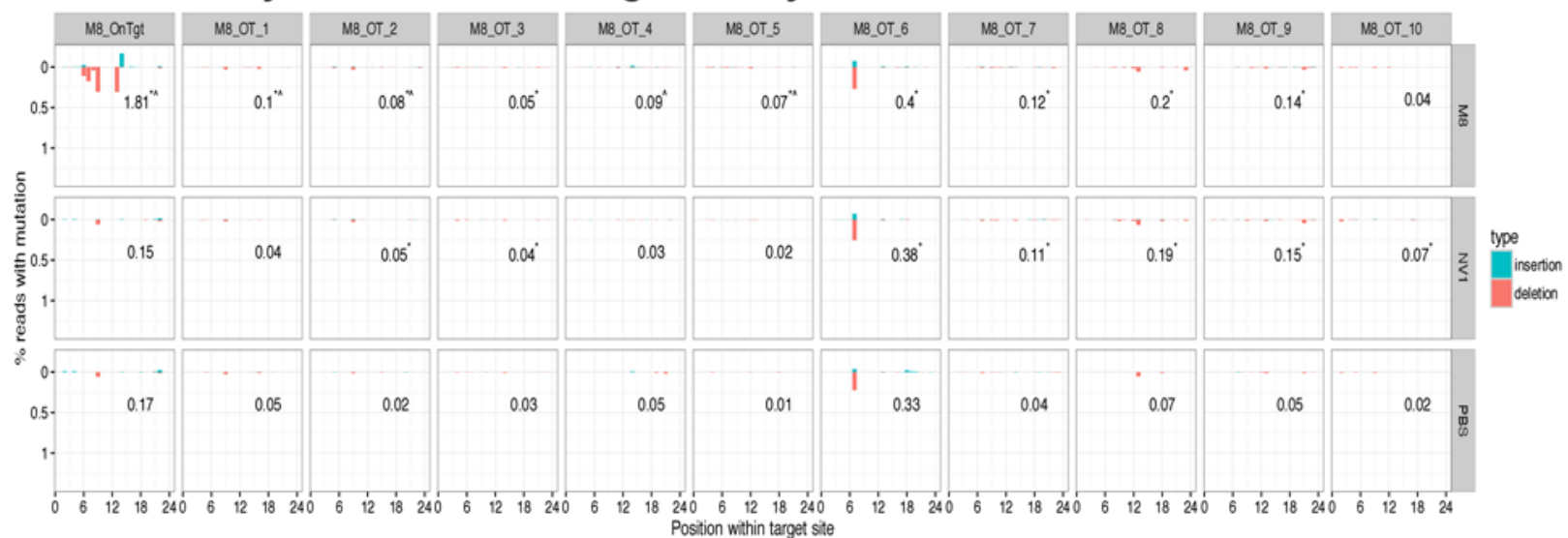

B NGS analysis of On and Off target activity of HSV1m5

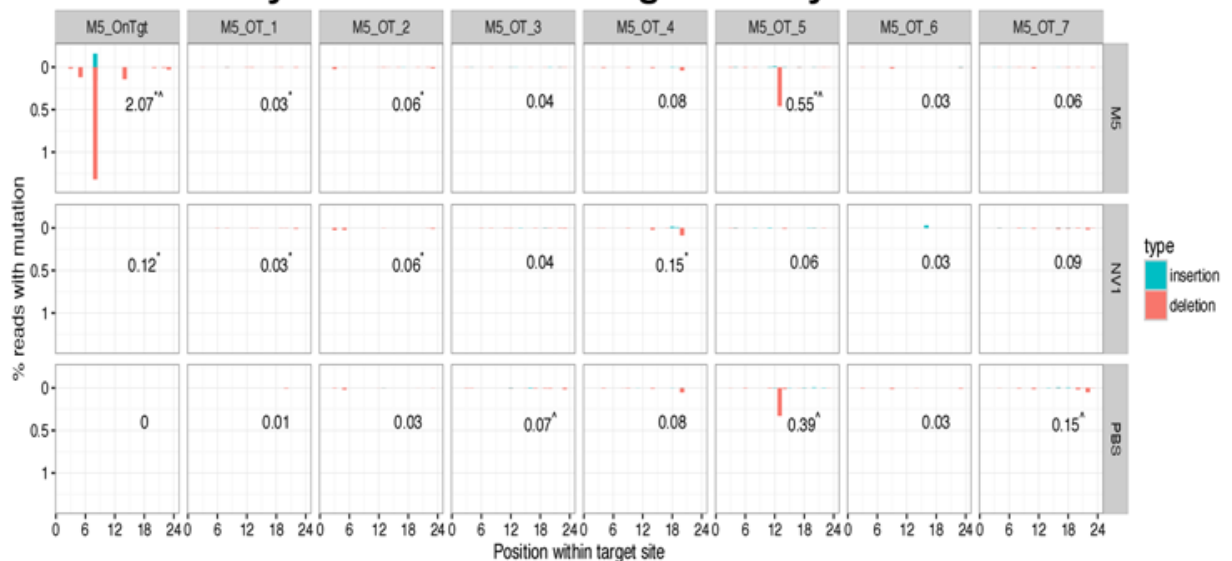

C NGS analysis of Off target activity of NV1

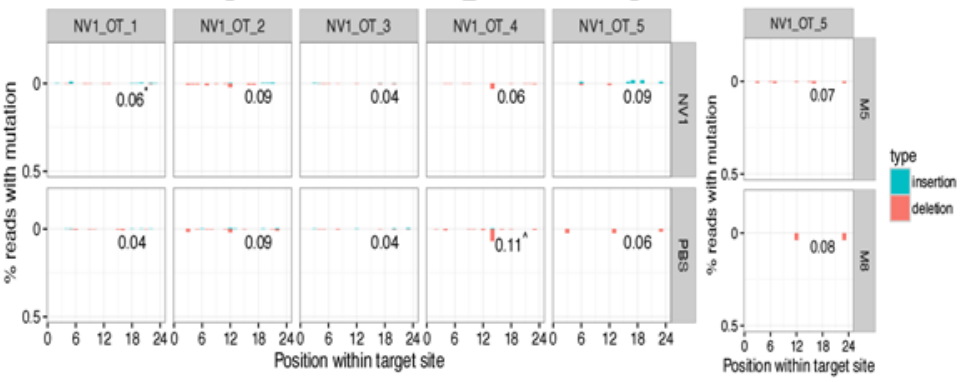

Figure 6. NGS analysis of HSV-specific HE off-target activity in vivo. Graphical representation of the percent mutations detected at the enzyme target site (On) and off-target sites (Off) for the HSV-specific enzymes HSV1m8 (A) and HSV1m5 (B), as well as the nonviral enzyme NV1 (C). The numbers in each plot indicate the total percent reads with mutation. The analysis was performed using TC genomic DNA from mice of the experiment described in Figure 5 and Tables 3-4: mouse 1 (PBS), mouse 2 (NV1), mouse 5 (HSV1m5), and mouse 10 (HSV1m8). Statistical analysis was performed using $\chi^{2}$ test of proportions. ${ }^{*} P \leq 0.05$ compared with PBS and ${ }^{\wedge} P \leq 0.05$ compared with NV1.

2-fold following TSA exposure of HE-treated neurons. It is unlikely that this resulted from a TSA-mediated effect on AAV transduction, since we previously found that HDACi exposure (including TSA) did not have any noticeable impact on the levels of AAV transduction when added prior to AAV exposure (13). In the present study, TSA was added after AAV transduction of the neuronal cultures, making it even less likely that the observed increase in mutagenesis was due to an indirect effect via AAV.

We have previously stressed the importance of efficient delivery as a determinant of the ultimate success of endonuclease therapy for viral infections $(11,28-30)$, and in the present study, we were able to deliver the enzymes to the sites of viral latency in a mouse model of latent HSV infection and reproducibly achieve endonuclease-mediated mutagenesis of viral genomes. Our work optimizing AAV delivery to the TG via whiskerpad injection of AAV1 into the whiskerpad (Dang et al., unpublished) suggests that this approach can lead to transduction of $25 \%-50 \%$ of TG neurons. In the experiments described here, endonuclease expression was detected in a smaller percentage of neurons $(5 \%-10 \%)$, perhaps due to less efficient detection of endonuclease compared with other marker proteins. Regardless, this level of endonuclease expression was sufficient to induce low but easily detectable mutation of latent HSV genomes. Furthermore, several treated animals showed evidence of delayed HSV reactivation from explanted TGs when 
A Experimental timeline

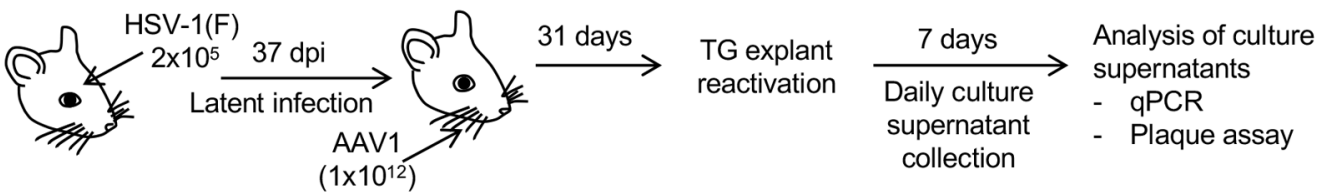

\section{B qPCR analysis of supernatants from TG explant cultures}
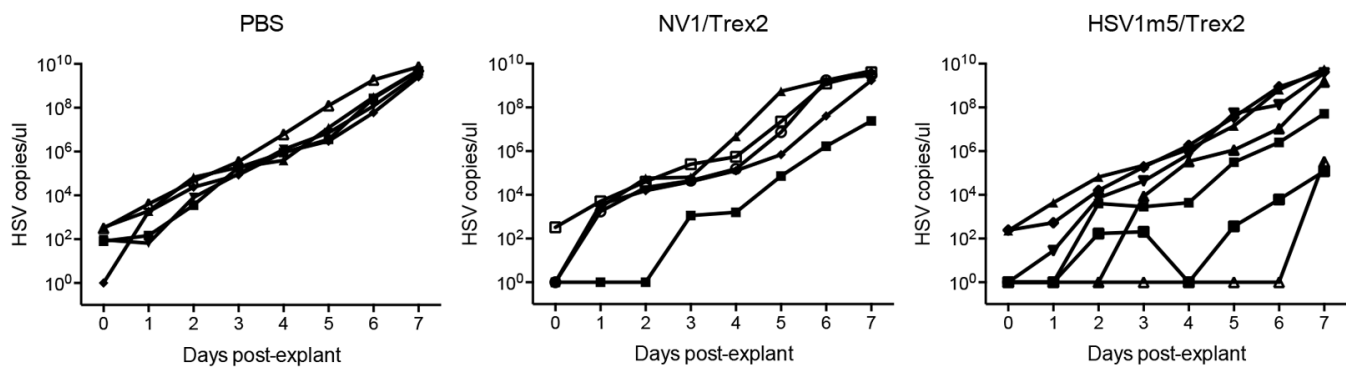

\section{Plaque assay analysis with supernatants from TG explant cultures}

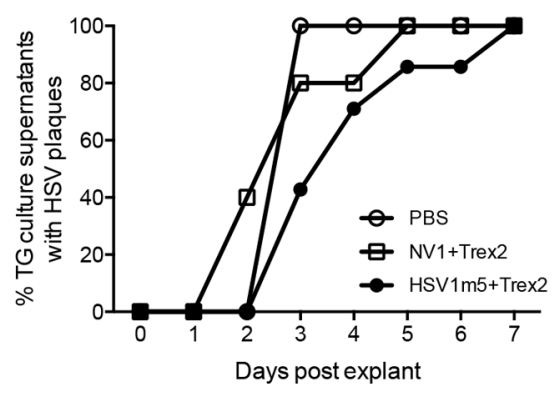

Figure 7. Impact of HE-directed mutagenesis of latent HSV on viral reactivation. (A) Experimental timeline for viral reactivation studies. Mice were infected with $2 \times 10^{5} \mathrm{PFU}$ HSV-1(F) in the right eye following corneal scarification and 37 days later, were injected in the right whiskerpad with $1 \times 10^{12}$ vector genomes of ssAAV1-smCBA-HSV1m5-Trex2mCherry or ssAAV1-smCBA-NV1-Trex2-mCherry. Individual ipsilateral TCs were explanted into culture wells containing monolayers of Vero cells, and culture media was collected and replaced daily for 7 days. (B) HSV DNA released into the culture media over the 7-day period was quantified by qPCR for control animals (PBS, left panel, $n=5$ ), animals treated with the nonviral enzyme (NV1/Trex2, middle panel, $n=5$ ), or animals treated with HSV-specific HE (HSV1m5/ Trex2, right panel, $n=7$ ). For each treatment group (PBS, NV1/Trex2, and HSV1m5/Trex2), 3 animals had no reactivable HSV and, therefore, were not depicted on the corresponding graph. Statistics using a log-rank test showed that the differences between controls and treated animals was not significant $(P=0.12)$. (C) Presence of infectious virus released into the culture media over the 7-day period was detected by plaque assay on Vero cell monolayers for control animals (PBS, left panel, $n=5$ ), animals treated with the nonviral enzyme (NV1/Trex2, middle panel, $n=5$ ), or animals treated with HSV-specific HE (HSV1m5/Trex2, right panel, $n=7)$. Statistics using a log-rank test showed that the differences between controls and treated animals was not significant $(P=0.2)$.

evaluated using 2 different assays (qPCR and plaque assay). While this did not reach statistical significance, it would be consistent with our previous in vitro findings that persistent endonuclease expression is particularly effective at inhibiting production of new virus from latently infected cells $(12,13)$. Clearly, future efforts should focus on increasing the efficiency of endonuclease delivery and target mutagenesis. Numerous strategies to increase endonuclease delivery efficiency are available, including evaluation of additional $\mathrm{AAV}$ serotypes and promoters, as well as investigation of alternative routes of administration. Additionally, nonviral approaches to endonuclease delivery warrant investigation, particularly approaches that use the same axonal transport mechanisms responsible for HSV and AAV transit from the periphery to the TG.

We evaluated a potential alternative outcome to HE-directed mutagenesis after cleavage of the target site in the HSV genome, DNA degradation, which would result in reduction of viral load after treatment. However, the experiments presented here, as well as our previously published work using in vitro latent infection (13), did not reveal a detectable loss of viral genomes. This has also been observed by others (25). DNA double strand breaks (DSBs) left unrepaired are potentially deleterious; therefore, cells have evolved 
robust repair of such lesions for the maintenance and propagation of genetic material (31). One potential way to force the infected cell to eliminate cleaved viral genomes would be to introduce 2 or more DSBs using a combination of targets or an enzyme that targets a site in the inverted repeats in the viral genome, as has been recently demonstrated for $\operatorname{HIV}(32,33)$.

Finally, our study addresses the issue of potential off-target effects in the context of an in vivo therapeutic use of endonuclease therapy. A genetic evaluation of on- versus off-target mutagenesis in treated animals was performed on samples obtained from the animals with the highest levels of on-target mutation. This analysis reveals negligible levels of off-target activity at the most closely related genomic sites. While encouraging, one caveat is that the apparent absence of off-target activity could be related to the low levels of on-target activity we observed, since the off-target activity of the endonucleases would presumably also depend on their cleavage efficiency. Our approach was also limited to analysis of in silico-predicted potential off-target sites and would not detect mutational events elsewhere in the host genome. It is also important to note that AAV delivery is not specific to HSV-infected cells; thus, a substantial number of uninfected cells were exposed to the enzymes and are consequently at risk for off-target activity. Despite these concerns, we are encouraged that mutation detected at off-target sites in treated animals was similar to background levels and that the treatment was well tolerated. It will be important in future studies to evaluate the off-target activity of endonucleases when a high degree of gene disruption is achieved at the intended enzyme target site and to observe treated animals for extended periods of time.

We and others have suggested that targeted endonucleases might represent a definitive curative approach to many chronic infections with DNA viruses, including HSV, Hepatitis B virus, HIV, human papillomavirus, and others $(11,28,30)$. Of these, HSV represents a particularly attractive target for several reasons. Unlike infections such as HIV, in which persistent genomes are maintained in cells scattered throughout the body, latent HSV genomes are restricted to neurons - specifically neuronal cell bodies of sensory and autonomic ganglia (34). Thus, delivery of endonucleases to the relevant cells, which is a critical determinant of ultimate success (11), is greatly simplified. Furthermore, the total number of cells containing persistent HSV is substantially less than in other viruses. A typical sensory ganglion contains on the order of $2 \times 10^{4}$ neurons (35), of which approximately $10 \%$ harbor HSV in an infected individual (36). Given that multiple ganglia are generally involved in clinical HSV infection, this would suggest that the total number of cells constituting the target population for a curative approach to HSV would be on the order of $2 \times 10^{4}$ neurons. This compares favorably with the situation in HIV infection, in which the reservoir likely consists of $1 \times 10^{7}$ or more memory $\mathrm{T}$ cells $(37,38)$, or $\mathrm{HBV}$, in which persistent infection is present in the great majority of the $2 \times 10^{11}$ hepatocytes in the human liver (29). Furthermore, unlike HIV and HBV, clinical benefit for HSV may require disruption of only a fraction of the persistent viral genomes. For example, cure of HIV has often been assumed to require complete elimination of all viral genomes, and even the more optimistic models suggest that clinically meaningful interventions would require a reduction by 2 logs (39) to 4 logs in replication-competent virus (40). In contrast, the frequency of HSV reactivation appears to be related to ganglionic viral load $(41,42)$, suggesting that reduction of the ganglionic viral load by one log or even less might provide substantial clinical benefit. Finally, in contrast to HIV or HBV, HSV has a higher fidelity of viral genome replication and relatively limited viral diversity, simplifying the development of endonucleases targeting all clinical isolates and minimizing the likelihood of viral escape, which has been demonstrated for HIV $(43,44)$.

The HSV-specific endonuclease used in the studies reported here was an HE (alternatively referred to as meganuclease), a class of targeted endonuclease that has received relatively less attention than the CRISPR/Cas system, zinc-finger nucleases (ZFNs), or TALENs. As we reviewed previously (11), meganucleases have certain characteristics that make them attractive as antiviral agents. Most notably, their relatively compact size allows packaging into a wide variety of delivery factors, which is especially important for AAV, given its limited packaging capacity. Furthermore, the relatively large area of protein/DNA contact for meganucleases, combined with the fact that - unlike other classes of endonucleases - DNA binding and cleavage are mediated by the same protein domain, suggests that meganucleases may have a superior specificity for their desired targets relative to off-target sites (45). However, rapid advancements in the other classes of endonucleases, particularly the CRISPR/Cas system, may favor these for future work. For example, development of smaller Cas9 proteins, such as those from Staphylococcus aureus or Neisseria meningitidis, now allows packaging into ssAAV vectors (46). In addition, optimization of single guide RNA (sgRNA) length (47) and the development of high-fidelity Cas9 variants $(48,49)$ now provide the CRISPR/ 
Cas system with a favorable on- versus off-targeting ratio.

The meganucleases used in this study are first-generation anti-HSV CreI derivatives and likely have less than ideal specificity for HSV relative to off-target sites. Thus, these first-generation endonucleases may represent a worst-case scenario in which to evaluate any possible undesired detrimental effects of endonuclease therapy. As such, it is encouraging to note that the administration of anti-HSV meganucleases to mice was well tolerated, with no evidence of neuronal loss or ganglionic inflammation. As improvements in endonuclease specificity continue, it is likely that the safety profile of this treatment approach will also continue to advance.

In summary, the data presented here provides critical proof-of-principle that targeted endonucleases can be delivered to relevant chronically infected cell populations in vivo, where they can induce mutation of latent virus. These results strongly support the continued development of targeted endonucleases as a potentially curative approach for HSV and other viral infections. Future work should seek to optimize endonuclease activity and specificity and to maximize the efficiency of enzyme delivery to the relevant cells in vivo. If sufficient advances can be made in these areas, the therapeutic efficacy and safety of this approach may well increase to the point that cures for HSV and other chronic viral infections can become a clinical reality.

\section{Methods}

Cells and herpesviruses. HEK293 (50) and Vero cell lines (ATCC CCL-81) were propagated in DMEM supplemented with $10 \%$ FBS. HSV-1 F $\Delta U_{s} 5$ is derived from HSV-1(F), replicates as WT parental HSV-1(F), and was described previously (51). This virus was used in the experiments presented in Figure 1D and Supplemental Figure 2 to confirm acute infection of the cultures as it expresses GFP during lytic infection, while GFP expression is switched off after establishing latent infection (13). HSV-1 F $\Delta U_{s} 5$ and HSV1(F) were propagated and titered on Vero cells.

$A A V$ vector plasmids. Plasmids pscAAV-sCMV-mCherry, pscAAV-sCMV-eGFP, pscAAV-CMV-eGFP have been described previously (43). pscAAV-smCBA-eGFP was generated by PCR amplification of the chicken $\beta$ actin (CBA) enhancer/promoter and a segment of the truncated CBA intron from the plasmid pCAGGS-FLPe (Gene Bridges) using primers smCBA-F: GAGATCGGCGCGCCAATTCGGTACCCTAGTTATTAATAGTAATCAATTAC and Intron-R: GAGATCCTCGAGATGCATGAACATGGTTAGCAGAGGCTCTAGCTCCCGGAGCCCTTTAAGGCTTTCAC, and cloned into the AscI/XhoI sites of pscAAV-EFS-pA (52) to generate pscAAV-smCBA-1. Then the mCherry gene fused to a segment of the CBA intron was amplified by PCR using primers Intron-F: GAGATCATGCATTCTTCTTTTTCCTACAGCTCCTGGGCAACGTGCTGGTTATTGTGCTGTCTCATCATTTTGGCAAAGGCGGCCGCGCCGCCACCATGGTGAGCAAGGGCGAGGAG, and mCherry-R2: GAGATCGGATCCGTCGACAAGCTTTTAATTAAGCGGCCGCTCACTTGTACAGCTCGTCCATGCCGCCGGT, and cloned into the NsiI/BamHI sites of pscAAV-smCBA-1 to generate pscAAV-smCBA-mCherry. pscAAV-smCBA-eGFP was produced by PCR amplifying the eGFP gene using primers NotI-GFP-F: GAGATCGCGGCCGCGCCGCCACCATGGTGAGCAAGGGCGAGGAGCTGT and NotI-GFP-R: GAGATCGCGGCCGCTTACTTGTACAGCTCGTCCATGCCG, and substituting eGFP with mCherry in pscAAV-smCBA-mCherry as a NotI fragment. pscAAV-sCMV-HSV1m5, pscAAV-sCMVHSV1m8, pscAAV-sCMV-NV1, and pscAAV-sCMV-Trex2 were generating by cloning sCMV sequences as a AscI-KpnI fragment from pscAAV-sCMV-eGFP into AscI-KpnI-digested pscAAV-CMV-HSV1m5, pscAAV-CMV-HSV1m8, pscAAV-CMV-NV1, or pscAAV-CMV-Trex2 (13). pssAAV-smCBA-HSV1m5Trex2-mCherry and pssAAV-smCBA-NV1-Trex2-mCherry were generated by PCR amplifying small CBA (smCBA)promoter from plasmid pscAAV-smCBA-eGFP with primers smCBA-F AATTAGTCAGCCATGAGCTTGGGGAATTCGGTACCCTAGTTATTAATAG and smCBA-R GGATATCCCATGGTGGCGGCGGTACCTTTGCCAAAATGATGAGACAGCAC and cloned into the AscI/KpnI of pscAAV-sCMV-HSV1m5 and pscAAV-sCMV-NV1.

Plasmid pssAAV-smCBA-HSV1m8-Trex2-mCherry was created by PCR amplifying the smCBA promoter from pssAAV-smCBA-HSV1m5-Trex2-mCherry using primers smCBA-F and smCBA-R (described above), PCR amplifying HSV1 8 from pscAAV-CMV-HSV1 8 using primers HSV1m8-F GTGCTGTCTCATCATTTTGGCAAAGGTACCGCCGCCACCATGGGAT ATCCATACGATGTCC and HSV1m8-R TCGACGTCACCGCATGTTAGAAGGGATCCTCTGCCCTCAGGAGAGGACTTTTTCTTCTCAGAG, and then PCR amplified smCBA and HSV1m8 were used to substitute CMV and HSV1m5 from 
pssAAV-CMV-HSV1m5-Trex2-mCherry digested with AscI/BamHI.

$A A V$ production and titering. AAV vectors of all serotypes were generated by transiently transfecting 293 cells using polyethylenimine (PEI) according to the method of Choi et al. (53). Briefly, HEK293 cells were transfected with a scAAV or ssAAV vector plasmid, a plasmid that expresses the AAV rep and capsid proteins, and a helper plasmid that expresses adenovirus helper proteins (pHelper). At 24 hours after transfection, media was changed to serum-free DMEM, and after 72 hours, cells were collected and resuspended in AAV lysis buffer (50mM Tris, $150 \mathrm{mM} \mathrm{NaCl}, \mathrm{pH} 8.5$ ) before freeze-thawing 4 times. AAV stocks were purified by iodixanol gradient separation $(53,54)$ followed by concentration into PBS using an Amicon Ultra-15 column (EMD Millipore) before storage at $-80^{\circ} \mathrm{C}$. All AAV vector stocks were quantified by qPCR using primers/probes against the AAV ITR, with linearized plasmid DNA as a standard, according to the method of Aurnhammer et al. (55). AAV stocks were treated with DNase I (Invitrogen) and Proteinase K (Sigma Aldrich) prior to quantification.

Establishment of neuronal cultures. Six- to10-week-old female Swiss Webster (Charles River Laboratories) mice were euthanized by $\mathrm{CO}_{2}$, and TG were harvested. Neuronal cultures were established after enzymatic digest as described by Bertke et al. (56) and after purification of the resulting cell homogenates using a percoll gradient as described (57). Neurons were counted and plated on either poly-D-lysine/laminincoated 8-well chamber slides (BD Biosciences) at a density of 3,000 neurons per well or poly-D-lysine/ laminin-coated $12 \mathrm{~mm}$ round slides (BD Biosciences) at a density of 4,000 neurons per well. Neurons were cultured without removing the nonneuronal cells that provide important growth support; therefore, these cultures contained a mixed population of neurons, satellite glial cells, and other cell types. Cultures were maintained with complete neuronal medium, consisting of Neurobasal A medium supplemented with $2 \%$ B27 supplement, 1\% PenStrep, L-glutamine (500 $\mu \mathrm{M})$, and nerve growth factor (NGF; $50 \mathrm{ng} / \mathrm{ml})$. Medium was replaced every 2-3 days with fresh medium. ACV $(100 \mathrm{nM})$ was added to the culture medium when TGs were harvested from HSV-1-infected mice.

$H S V$ infection and AAV inoculation of mice. Mice were housed in accordance with the institutional and NIH guidelines on the care and use of animals in research. Female Swiss Webster mice (Charles River Laboratories) 6-8 weeks old were used for all studies. For ocular HSV infection, mice anesthetized by i.p. injection of ketamine $(100 \mathrm{mg} / \mathrm{kg})$ and xylazine $(12 \mathrm{mg} / \mathrm{kg})$ were infected with $2 \times 10^{5} \mathrm{PFU}$ of HSV1(F) following corneal scarification of the right eye using a 28-gauge needle. For AAV inoculation, mice anesthetized with ketamine/xylazine were injected intradermally in the right whiskerpad with the indicated AAV vector dose in a $50 \mu 1$ volume. All animals were infected with HSV and injected with AAV on only their right ipsilateral side.

Ex vivo HSV reactivation. Ipsilateral TG were collected at the time indicated and placed into individual culture wells containing a monolayer of Vero cells. Culture media from each well were collected daily over a 7-day period. For each TG at each time point, half of the medium was used for qPCR quantification of HSV DNA released into the culture supernatants, and the other half was placed into individual culture wells containing monolayers of Vero cells to detect the release of infectious virus.

HSV on- and off-target site PCR amplification. Total genomic DNA (gDNA) was extracted using either the DNeasy Tissue and Blood micro kit (Qiagen) for neuronal cultures or the DNeasy Tissue and Blood mini kit (Qiagen) for whole TGs. Platinum $P f x$ DNA polymerase (Invitrogen) and 2-5 $\mu 1$ of gDNA were used to PCR amplify the regions containing the HSV1m5 or HSV1m8 on-target sites. $\mathrm{U}_{\mathrm{L}} 19 \mathrm{~B}$ primers (forward 5'-GGCCGG CGGAAGTAGTTGAC-3' and reverse 5'-CACCGACATGGGCAACCTTC-3') or $\mathrm{U}_{\mathrm{L}} 30$ primers (forward 5'-GAGAACGTGGAGCACGCGTACGGC-3' and reverse 5'-GGCCCGGTTTGAGACGGTACCAGC-3') were used with thermocycler conditions of $94^{\circ} \mathrm{C}$ for 5 minutes, $40-45$ cycles $\left(94^{\circ} \mathrm{C}\right.$ for 30 seconds, $60^{\circ} \mathrm{C}$ for 30 seconds, $70^{\circ} \mathrm{C}$ for 30 seconds), and then $70^{\circ} \mathrm{C}$ for 5 minutes.

The off-target predictor program PROGNOS was used and adapted to determine the closest potential off-target sites in the mouse genome (58). Phusion DNA polymerase (New England Biolabs) was used to PCR amplify the regions containing the HSV1m5, HSV1m8, and NV1 off-target sites. The primers are listed in Supplemental Table 3 with the following thermocycler conditions: $98^{\circ} \mathrm{C} 30$ seconds, 40 cycles $\left(98^{\circ} \mathrm{C}\right.$ 10 seconds, $65^{\circ} \mathrm{C} 20$ seconds, $72^{\circ} \mathrm{C} 15$ seconds), and then $72^{\circ} \mathrm{C} 5$ minutes. Up to 10 of the off-target sites for each enzyme with the lowest number of differences ( 6 or fewer mismatches) were retained if the region containing the off-target site was amplified by PCR in analyzed samples.

T7E1 assay. The T7E assay was performed as follows. After PCR amplification of target site from HSV genomes, followed by purification using Zymo Research clean and concentrator-5 kit (Zymo Research), $300-400$ ng of DNA amplicon was denatured for 10 minutes at $95^{\circ} \mathrm{C}$ and slowly reannealed by cooling 
down to room temperature. DNA was then digested with 5 units of T7E1 (New England Biolabs) for 30 minutes at $37^{\circ} \mathrm{C}$ and resolved in a $3 \%$ agarose gel. Quantification to determine the levels of gene disruption was performed using ImageJ software $(\mathrm{NIH}$, ref. 59)and calculated using the formula: $100 \times(1-[1-$ fraction cleaved $\left.]^{1 / 2}\right)$, where fraction cleaved $=$ the density of cleavage product/(density of cleavage product + density of uncleaved product).

PCR amplicon sequencing. PCR amplicons containing the target regions generated as described above were cleaned using ZymoResearch clean and concentrator-5 kit and cloned into the PCR Blunt-TOPO vector using the Zero Blunt TOPO PCR cloning kit (Invitrogen). Target sequence analysis was performed by bacterial colony sequencing Genewiz.

ddPCR quantification. Viral genome quantification by ddPCR was performed as described previously (13) using an AAV ITR primer/probe set for AAV (55) and a gB primer/probe set for HSV (13).

Illumina NGS. NGS of HE target sites and off-target sites was performed using PCR products generated with the target site-specific primers, described above and in Supplemental Table 3, and a MiSeq sequencer (Illumina), as previously described $(43,60)$. The sequencing data has been archived in the NIH Short Read Archive under the following accession numbers: SAMN05417676 for data in Table 2 and Supplemental Table 1, SAMN05417677 for data in Table 3 and 4, and SAMN05417686 for the off-target sequence analysis in Figure 6.

$I H C$. TG were fixed in 10\% neutral buffered formalin, paraffin embedded, and then sectioned at $4 \mu \mathrm{m}$ thickness. For mCherry staining, antigen retrieval was performed for 12 hours at $\mathrm{pH} 9$ in a $65^{\circ} \mathrm{C}$ water bath using Tris-EDTA buffer. Sections were then stained with a mouse monoclonal anti-mCherry antibody $(1: 1,000)$ provided by FHCRC Experimental Histopathology (Benjamin Hoffstrom), and detection was performed using PowerVision poly-HRP anti-mouse (Leica), along with the chromagen DAB (Dako) 2 times for 4 minutes. Slides were then counterstained with hematoxylin (BioCare) and Tacha's bluing solution at $25 \%$ for 2 minutes. Isotype controls of mouse Ig were used as negative control. For NeuN staining, antigen retrieval was performed as above for 12 hours at $\mathrm{pH} 9$ in a $65^{\circ} \mathrm{C}$ water bath. Sections were then stained with a mouse monoclonal anti-NeuN antibody (clone A60, Millipore mab377, 1:800), and detection was performed using PowerVision poly-HRP anti-rabbit and the Mouse on Mouse Basic Kit (Vector Laboratories, catalog BMK-2202).

Microscopy. Cells were imaged at the indicated magnification using a TE2000 microscope (Nikon) equipped with a CDD camera (CoolSNAP ES, Photometrics) and Metavue software (Universal imaging).

DAB-stained bright field images were scanned at $40 \times$ (Aspera) and captured at fixed exposure and position using ImageScope software.

Fluorescence quantification. Levels of reporter gene expression from the different promoters were measured by quantification of GFP fluorescence using Image J software (NIH, ref. 59) and 16-bit images after applying a threshold using the ReniEntropy method. The corrected total fluorescence (CTF) was calculated as the integrated density of ROI: (area of ROI $\times$ mean fluorescence of background readings).

Statistics. Comparison of the virus titer from HE-treated and control cells was made using 1-sided $t$ test in Figure 1 and Supplemental Figure 2. In Figure 6, we performed a $\chi^{2}$ test of proportions to compare the target mutation frequency for each sample from HE-treated animals against the frequency obtained with samples from animals treated with NV1 and PBS. The prop.test() function was used in R with the Yates continuity correction, and 1-sided $P$ values were computed to determine whether mutation rates in HE-treated samples were significantly higher than NV1- and PBS-treated samples. In Figure 7, a logrank test was performed to determine whether controls and treated animals in the in vivo reactivation experiment differed in time to viral detection. A $P$ value less than 0.05 was considered significant in all the statistical analysis.

Study approval. All animal procedures were approved by the Institutional Animal Care and Use Committee of the Fred Hutchinson Cancer Research Center.

\section{Author Contributions}

KRJ and MA conceived and designed the studies and analyzed data. MA, EAM, and ALG conducted experiments and acquired and analyzed data. ML, HSDF, LS, NN, and TN conducted experiments and acquired data. MLH designed experiments. PR performed the NGS data analysis. DS designed and generated AAV vectors. AM conducted the statistical analyses for the in vivo experiments. RG provided plasmids with the endonuclease genes. MA and KRJ wrote the manuscript. 


\section{Acknowledgments}

We thank Shared Resources and Experimental Histopathology at Fred Hutchinson Cancer Research Center for technical support. We also thank Daniel Strongin for critical reading of this manuscript. This work was funded by a philanthropic grant from the Caladan Foundation and in part by NIH grant R21 AI117519. This work was funded by a philanthropic grant from the Caladan Foundation (PI: K.R. Jerome), and in part by NIH grant R21 AI117519 (PI: K.R. Jerome). E.A. Madden was supported under NCI grant 3PC30CA015704 (PI: G. Gilliland, FHCRC).

Address correspondence to: Keith R. Jerome, Fred Hutchinson Cancer Research Center, 1100 Fairview Avenue N, E5-110, Seattle, Washington 98109, USA. Phone: 206.667.6793; E-mail: kjerome@fredhutch.org.

1. Roizman B, Knipe DM, Whitley RJ. 2007. Herpes Simplex Viruses. In: Knipe DM, Howley PM, eds. Fields Virology. Wolters Kluwer Health/Lippincott Williams \& Wilkins; 2007:2502-2599.

2. Wald A, Zeh J, Selke S, Ashley RL, Corey L. Virologic characteristics of subclinical and symptomatic genital herpes infections. N Engl J Med. 1995;333(12):770-775.

3. Crespi CM, Cumberland WG, Wald A, Corey L, Blower S. Longitudinal study of herpes simplex virus type 2 infection using viral dynamic modelling. Sex Transm Infect. 2007;83(5):359-364.

4. Wald A, Corey L, Cone R, Hobson A, Davis G, Zeh J. Frequent genital herpes simplex virus 2 shedding in immunocompetent women. Effect of acyclovir treatment. J Clin Invest. 1997;99(5):1092-1097.

5. Fife KH, et al. Effect of valacyclovir on viral shedding in immunocompetent patients with recurrent herpes simplex virus 2 genital herpes: a US-based randomized, double-blind, placebo-controlled clinical trial. Mayo Clin Proc. 2006;81(10):1321-1327.

6. Gupta R, et al. Valacyclovir and acyclovir for suppression of shedding of herpes simplex virus in the genital tract. J Infect Dis. 2004;190(8):1374-1381.

7. Mertz GJ, et al. Long-term acyclovir suppression of frequently recurring genital herpes simplex virus infection. A multicenter double-blind trial. JAMA. 1988;260(2):201-206.

8. Wald A, Zeh J, Barnum G, Davis LG, Corey L. Suppression of subclinical shedding of herpes simplex virus type 2 with acyclovir. Ann Intern Med. 1996;124(1 Pt 1):8-15.

9. Johnston C, et al. Standard-dose and high-dose daily antiviral therapy for short episodes of genital HSV-2 reactivation: three randomised, open-label, cross-over trials. Lancet. 2012;379(9816):641-647.

10. Corey L, et al. Once-daily valacyclovir to reduce the risk of transmission of genital herpes. N Engl J Med. 2004;350(1):11-20.

11. Schiffer JT, Aubert M, Weber ND, Mintzer E, Stone D, Jerome KR. Targeted DNA mutagenesis for the cure of chronic viral infections. J Virol. 2012;86(17):8920-8936.

12. Grosse S, et al. Meganuclease-mediated Inhibition of HSV1 Infection in Cultured Cells. Mol Ther. 2011;19(4):694-702.

13. Aubert M, et al. In vitro Inactivation of Latent HSV by Targeted Mutagenesis Using an HSV-specific Homing Endonuclease. Mol Ther Nucleic Acids. 2014;3:e146.

14. Samaranch L, et al. Strong cortical and spinal cord transduction after AAV7 and AAV9 delivery into the cerebrospinal fluid of nonhuman primates. Hum Gene Ther. 2013;24(5):526-532.

15. Hirai T, et al. Intrathecal AAV serotype 9-mediated delivery of shRNA against TRPV1 attenuates thermal hyperalgesia in a mouse model of peripheral nerve injury. Mol Ther. 2014;22(2):409-419.

16. Jacques SJ, et al. AAV8(gfp) preferentially targets large diameter dorsal root ganglion neurones after both intra-dorsal root ganglion and intrathecal injection. Mol Cell Neurosci. 2012;49(4):464-474.

17. Xu Q, et al. In vivo gene knockdown in rat dorsal root ganglia mediated by self-complementary adeno-associated virus serotype 5 following intrathecal delivery. PLoS One. 2012;7(3):e32581.

18. Mason MR, et al. Comparison of AAV serotypes for gene delivery to dorsal root ganglion neurons. Mol Ther. 2010;18(4):715724.

19. McCarty DM, Monahan PE, Samulski RJ. Self-complementary recombinant adeno-associated virus (scAAV) vectors promote efficient transduction independently of DNA synthesis. Gene Ther. 2001;8(16):1248-1254.

20. McCarty DM, Fu H, Monahan PE, Toulson CE, Naik P, Samulski RJ. Adeno-associated virus terminal repeat (TR) mutant generates self-complementary vectors to overcome the rate-limiting step to transduction in vivo. Gene Ther. 2003;10(26):21122118.

21. Muñoz IG, et al. Molecular basis of engineered meganuclease targeting of the endogenous human RAG1 locus. Nucleic Acids Res. 2011;39(2):729-743.

22. Sawtell NM, Poon DK, Tansky CS, Thompson RL. The latent herpes simplex virus type 1 genome copy number in individual neurons is virus strain specific and correlates with reactivation. $J$ Virol. 1998;72(7):5343-5350.

23. Leib DA, et al. A deletion mutant of the latency-associated transcript of herpes simplex virus type 1 reactivates from the latent state with reduced frequency. J Virol. 1989;63(7):2893-2900.

24. Cliffe AR, Coen DM, Knipe DM. Kinetics of facultative heterochromatin and polycomb group protein association with the herpes simplex viral genome during establishment of latent infection. MBio. 2013;4(1):1.

25. Roehm PC, et al. Inhibition of HSV-1 Replication by Gene Editing Strategy. Sci Rep. 2016;6:23146

26. Knipe DM, Cliffe A. Chromatin control of herpes simplex virus lytic and latent infection. Nat Rev Microbiol. 2008;6(3):211-221.

27. Terry-Allison T, Smith CA, DeLuca NA. Relaxed repression of herpes simplex virus type 1 genomes in Murine trigeminal neurons. J Virol. 2007;81(22):12394-12405.

28. Weber ND, Aubert M, Dang CH, Stone D, Jerome KR. DNA cleavage enzymes for treatment of persistent viral infections: 
recent advances and the pathway forward. Virology. 2014;454-455:353-361.

29. Schiffer JT, Swan DA, Stone D, Jerome KR. Predictors of hepatitis B cure using gene therapy to deliver DNA cleavage enzymes: a mathematical modeling approach. PLoS Comput Biol. 2013;9(7):e1003131.

30. Stone D, Kiem HP, Jerome KR. Targeted gene disruption to cure HIV. Curr Opin HIV AIDS. 2013;8(3):217-223.

31. Hartlerode AJ, Scully R. Mechanisms of double-strand break repair in somatic mammalian cells. Biochem J. 2009;423(2):157168.

32. Kaminski R, et al. Elimination of HIV-1 Genomes from Human T-lymphoid Cells by CRISPR/Cas9 Gene Editing. Sci Rep. 2016;6:22555

33. Qu X, et al. Zinc-finger-nucleases mediate specific and efficient excision of HIV-1 proviral DNA from infected and latently infected human T cells. Nucleic Acids Res. 2013;41(16):7771-7782.

34. Lee S, Ives AM, Bertke AS. Herpes Simplex Virus 1 Reactivates from Autonomic Ciliary Ganglia Independently from Sensory Trigeminal Ganglia To Cause Recurrent Ocular Disease. J Virol. 2015;89(16):8383-8391.

35. LaGuardia JJ, Cohrs RJ, Gilden DH. Numbers of neurons and non-neuronal cells in human trigeminal ganglia. Neurol Res. 2000;22(6):565-566

36. Wang K, Lau TY, Morales M, Mont EK, Straus SE. Laser-capture microdissection: refining estimates of the quantity and distribution of latent herpes simplex virus 1 and varicella-zoster virus DNA in human trigeminal Ganglia at the single-cell level. $J$ Virol. 2005;79(22):14079-14087.

37. Chun TW, et al. Relationship between the size of the human immunodeficiency virus type 1 (HIV-1) reservoir in peripheral blood CD4+ T cells and CD4+:CD8 + T cell ratios in aviremic HIV-1-infected individuals receiving long-term highly active antiretroviral therapy. J Infect Dis. 2002;185(11):1672-1676.

38. Chun TW, et al. Quantification of latent tissue reservoirs and total body viral load in HIV-1 infection. Nature. 1997;387(6629):183-188.

39. Conway JM, Perelson AS. Residual Viremia in Treated HIV+ Individuals. PLoS Comput Biol. 2016;12(1):e1004677.

40. Hill AL, Rosenbloom DI, Fu F, Nowak MA, Siliciano RF. Predicting the outcomes of treatment to eradicate the latent reservoir for HIV-1. Proc Natl Acad Sci U S A. 2014;111(37):13475-13480.

41. Hoshino Y, Pesnicak L, Cohen JI, Straus SE. Rates of reactivation of latent herpes simplex virus from mouse trigeminal ganglia ex vivo correlate directly with viral load and inversely with number of infiltrating CD8+ T cells. J Virol. 2007;81(15):8157-8164.

42. Sawtell NM, Poon DK, Tansky CS, Thompson RL. The latent herpes simplex virus type 1 genome copy number in individual neurons is virus strain specific and correlates with reactivation. $J$ Virol. 1998;72(7):5343-5350.

43. De Silva Feelixge HS, et al. Detection of treatment-resistant infectious HIV after genome-directed antiviral endonuclease therapy. Antiviral Res. 2016;126:90-98.

44. Wang G, Zhao N, Berkhout B, Das AT. CRISPR-Cas9 Can Inhibit HIV-1 Replication but NHEJ Repair Facilitates Virus Escape. Mol Ther. 2016;24(3):522-526.

45. Stoddard BL. Homing endonucleases: from microbial genetic invaders to reagents for targeted DNA modification. Structure. 2011;19(1):7-15.

46. Kennedy EM, Cullen BR. Bacterial CRISPR/Cas DNA endonucleases: A revolutionary technology that could dramatically impact viral research and treatment. Virology. 2015;479-480:213-220.

47. Fu Y, Sander JD, Reyon D, Cascio VM, Joung JK. Improving CRISPR-Cas nuclease specificity using truncated guide RNAs. Nat Biotechnol. 2014;32(3):279-284.

48. Kleinstiver BP, et al. High-fidelity CRISPR-Cas9 nucleases with no detectable genome-wide off-target effects. Nature. 2016;529(7587):490-495.

49. Slaymaker IM, Gao L, Zetsche B, Scott DA, Yan WX, Zhang F. Rationally engineered Cas9 nucleases with improved specificity. Science. 2016;351(6268):84-88.

50. Graham FL, Smiley J, Russell WC, Nairn R. Characteristics of a human cell line transformed by DNA from human adenovirus type 5. J Gen Virol. 1977;36(1):59-74.

51. Aubert M, et al. The antiapoptotic herpes simplex virus glycoprotein J localizes to multiple cellular organelles and induces reactive oxygen species formation. J Virol. 2008;82(2):617-629.

52. Weber ND, et al. AAV-mediated delivery of zinc finger nucleases targeting hepatitis B virus inhibits active replication. PLoS One 2014;9(5):e97579.

53. Choi VW, Asokan A, Haberman RA, Samulski RJ. Production of recombinant adeno-associated viral vectors for in vitro and in vivo use. Curr Protoc Mol Biol. 2007; Chapter 16:Unit 16.25.

54. Zolotukhin S, et al. Recombinant adeno-associated virus purification using novel methods improves infectious titer and yield. Gene Ther. 1999;6(6):973-985.

55. Aurnhammer C, et al. Universal real-time PCR for the detection and quantification of adeno-associated virus serotype 2-derived inverted terminal repeat sequences. Hum Gene Ther Methods. 2012;23(1):18-28.

56. Bertke AS, Swanson SM, Chen J, Imai Y, Kinchington PR, Margolis TP. A5-positive primary sensory neurons are nonpermissive for productive infection with herpes simplex virus 1 in vitro. J Virol. 2011;85(13):6669-6677.

57. Malin SA, Davis BM, Molliver DC. Production of dissociated sensory neuron cultures and considerations for their use in studying neuronal function and plasticity. Nat Protoc. 2007;2(1):152-160.

58. Fine EJ, Cradick TJ, Zhao CL, Lin Y, Bao G. An online bioinformatics tool predicts zinc finger and TALE nuclease off-target cleavage. Nucleic Acids Res. 2014;42(6):e42.

59. Schneider CA, Rasband WS, Eliceiri KW. NIH Image to ImageJ: 25 years of image analysis. Nat Methods. 2012;9(7):671-675

60. Sedlak RH, et al. Digital detection of endonuclease mediated gene disruption in the HIV provirus. Sci Rep. 2016;6:20064. 